\title{
Sequential interferometric techniques for measuring independent values of the refractive index and material thickness of semiconductor wafers
}

\author{
Glen D. Gillen \\ Air Force Research Laboratory, Materials and Manufacturing Directorate, Anteon Corporation, WPAFB, OH 45433 \\ Voice: 937 252-3132, Fax: 937 252-0418,Email:ggillen@Anteon.com \\ Shekhar Guha \\ Air Force Research Laboratory, Materials and Manufacturing Directorate, WPAFB, OH 45433
}

\begin{abstract}
A technique is presented using both Michelson and Fabry-Perot interferometry to independently measure the refractive index and the material thickness of semiconductor wafers. The method does not require accurate prior knowledge of either quantity.
\end{abstract}

The refractive index, $n$, of materials is often determined by interferometrically measuring the phase change that light undergoes in passing through a plane parallel slab of the material $[1,2]$. Since the phase change depends upon the value of $n$ as well as the slab thickness, $d$, in order to obtain accurate values of $n$, using this method, it is important to know $d$ accurately. Fabry-Perot etalon interferometry has been used to optically measure $d$ [3], but the precision of thickness measurements is limited by the precision of the known refractive index value. This method is advantageous for materials in experimental conditions where both $n$ and $d$ are changing and direct physical measurement of $d$ is difficult.

We will demonstrate that the Michelson and the Fabry-Perot interferometric methods can be used sequentially to determine independent and absolute values of both the material's thickness and the material's refractive index over a wide range of temperatures of practical interest. The method does not require that either quantity be initially well known. First, using data from both experiments the material's physical thickness is determined. Then the thickness value is used to determine the material's refractive index using either of the interferometric methods. We present experimental verification of this method by measuring $n$ and $d$ for a range of common infrared materials at both room temperature and cryogenic temperatures.

If a flat-parallel plate is inserted into one arm of a Michelson interferometer and rotated about an axis perpendicular to the laser beam propagation path, the angle dependent phase term of the observed intensity exiting the interferometer is given by [4]

$$
\phi_{m}(\theta)=\left[\frac{4 \pi d}{\lambda}\left(\sqrt{n^{2}-\sin ^{2} \theta}+1-\cos \theta\right)\right],
$$




\section{OTuD4}

where $d$ is the wafer thickness, $\lambda$ is the wavelength of the light, $n$ is the refractive index of the wafer and $\theta$ is the angle of incidence. Similarly, the phase dependent term of the transmitted intensity of a wafer rotated in a FabryPerot etalon interferometer can be written as [3]

$$
\phi_{f p}(\theta)=\frac{4 \pi d}{\lambda}\left(\sqrt{n^{2}-\sin ^{2} \theta}\right)
$$

Using phase information from both interferometric experiments, the absolute thickness of the wafer can be determined from the difference between equations (1) and (2), independent of the value of $n$. Finally, using the determined value of $d$, the refractive index can be extracted from the raw data from either interferometric technique.

We will present experimental verification of this method for infrared semiconductor wafers of GaAs, Ge, Si, and $\mathrm{ZnSe}$ at room temperature and cryogenic temperatures. Using this method refractive index values with uncertainties in the third decimal place, and thickness measurements accurate to within 5 microns are obtained.

[1] C. A. Proctor, "Index of refraction and dispersion with the interferometer," Phys. Rev. 24, 195-201 (1907).

[2] J. F. H. Nichols, B. Henderson, and B. H. T. Chai, "Accurate determination of the indices of refraction of nonlinear optical crystals," Appl. Opt. 36, 8587-8594 (1997).

[3] John C. Brasunas and G. Mark Curshman, "Interferometric but nonspectroscopic technique for measuring the thickness of a transparent plate," Opt. Eng. 34, 2126-2130 (1995).

[4] Glen D. Gillen and Shekhar Guha, "Refractive-index measurements of zinc germanium diphosphide at 300 and $77 \mathrm{~K}$ by use of a modified Michelson interferometer," Appl. Opt. 43, 2054-2058 (2004). 